\title{
Synthesis and Cyclodehydration of Hydroxyphenols: A New Stereoselective Approach to 3-Aryl-2,3- Dihydrobenzofuranes
}

Ferruccio Bertolini, Paolo Crotti, Valeria Di Bussolo, Franco Macchia, and Mauro Pineschi*.

Dipartimento di Chimica Bioorganica e Biofarmacia, Università di Pisa, Via Bonanno 33, 56126 Pisa, Italy. pineschi@farm.unipi.it

\section{List of contents}

- Indication of Material and Methods used (page S2).

- Text giving all experimental procedures and characterization of new hydroxyphenols of type 3 (page S3-S11)

- Text giving all experimental procedures and characterization of new 2,3dihydrobenzofuranes of type 4 (cis) and 5 (trans) reported in the main text (page S11-S16)

- Additional results of cyclodehydratation of hydroxyphenols to 2,3dihydrobenzofuranes (Table 3) (page S17)

- Text giving all experimental procedures and characterization of new compounds of type 4 and 5 not reported in the main text (page S18-S20) 
General Methods. Diethylazadicarboxylate, triphenylphosphine, the solution of $\mathrm{BH}_{3} \cdot \mathrm{Me}_{2} \mathrm{~S}$, and all phenols employed in this study were used as received. Anhydrous THF, $\mathrm{CH}_{2} \mathrm{Cl}_{2}$ (containing molecolar sieves) were purchased and used as such. Analytical TLC were performed on Alugram SIL G/UV254 silica gel sheets with detection by $0.5 \%$ Phosphomolybdic acid solution in 95\% EtOH. Silica gel 60 was used for flash chromatography. Solvents for extraction and chromatography were HPLC grade.

${ }^{1} \mathrm{H}$ NMR spectra were recorded at 200 or $250 \mathrm{MHz}$. Chemical shifts are reported in ppm downfield from tetramethylsilane with the solvent resonance as the internal standard (deuterochloroform: $\delta$ 7.26, deuteromethanol: $\delta$ 3.31). ${ }^{13} \mathrm{C}$ NMR spectra were recorded at $50 \mathrm{MHz}$ or at $62.5 \mathrm{MHz}$ with complete proton decoupling. Chemical shifts are reported in ppm downfield from tetramethylsilane with the solvent resonance as the internal standard (deuterochloroform: $\delta$ 77.0, deuteromethanol: $\delta$ 49.0).

1D NOESY spectrum was performed on a spectrometer operating at $600 \mathrm{MHz}$ for $1 \mathrm{H}$. The selective 1D NOE spectrum was acquired with 128 scans in 32K data points with a $15 \mathrm{~s}$ relaxation delay and a mixing time of $1.6 \mathrm{~s}$ by using selective pulse generated by means of an appropriate software.

Analytical high performance liquid chromatography (HPLC) were performed on a column with $0.5 \mathrm{~mL}$ solvent flow and detection at $254 \mathrm{~nm}$.

GC/MS spectra were obtained operating at $70 \mathrm{eV}$.

Elemental analyses were performed at the Department of Pharmaceutical Sciences of the University of Pisa. 
<smiles>Cc1cc(C)c(C(c2ccccc2)[C@H](C)O)c(O)c1</smiles>

2-((1R*, 2S*)-2-Hydroxy-1-phenylpropyl)-3,5-dimethylphenol (3ab) (Table 1, entry 1).

Using the general procedure, a solution of tris-(3,5-dimethylphenyl) borate (1b) $(560.7 \mathrm{mg}, 1.5 \mathrm{mmol})$ in $\mathrm{CH}_{2} \mathrm{Cl}_{2}(1.0 \mathrm{~mL})$ was added at $78^{\circ} \mathrm{C}$ under argon to a solution of trans- $\beta$-methylstyrene oxide (2a) hours at $-40^{\circ} \mathrm{C}$. The crude mixture, containing $35 \%$ of $\mathrm{O}$-alkylated product, was purified by column chromatography eluting with hexanes/AcOEt 85:15 to give compound 3ab (115.2 mg, 45\%), as a white solid. M.p $=72-75^{\circ} \mathrm{C}$.

${ }^{1} \mathrm{H}$ NMR (200 MHz, $\mathrm{CDCl}_{3}$ ) $\delta 1.29$ (d, 3H, J=6.6 Hz); 2.21 (s, 3H); 2.29 (s, 3H); 4.40 (d, 1H, J= $4.1 \mathrm{~Hz}$ ); 4.59- 4.75 (m, 1H); 6.60 (br, s, 1H); 6.70 (br, s, 1H); 7.14$7.21(\mathrm{~m}, 5 \mathrm{H})$.

${ }^{13} \mathrm{C}$ NMR (50 MHz, $\left.\mathrm{CDCl}_{3}\right) \delta$ 20.5, 20.8, 21.1, 51.4, 70.9, 116.6, 123.3, 126.6, 128.2, 128.7, 137.9, 138.6, 139.1, 140.2, 155.2.

Anal. Calcd. For $\mathrm{C}_{17} \mathrm{H}_{20} \mathrm{O}_{2}$ : C, 79.65\%; H, 7.86\%. Found: C, 79.41\%; H, 7.53\%.<smiles>Cc1cc(C)cc(OC(c2ccccc2)[C@H](C)O)c1</smiles>
(1S*, 2S*)-1-(3,5-Dimethylphenoxy)-1-phenylpropan-2-ol. The first eluting fractions of the above described column chromatography afforded pure (1S*, 2S*)-1-phenyl-1-(3,5dimethylphenoxy)-propan-2-ol, as a white solid (yield= 21\%). $\mathrm{M} \cdot \mathrm{p}=134-136^{\circ} \mathrm{C}$.

${ }^{1} \mathrm{H}$ NMR (200 MHz, CDCl ${ }_{3}$ ) $\delta 1.09$ (d, 3H, J=6.4 Hz); 2.19 (s, 6H); $2.85 \mathrm{OH}$ (br, s, 1H); 4.00-4.10 (m, 1H); 4.82 (d, 1H, J=7.3 Hz); 6.44-6.53 (m, 3H); 7.20-7.40 (m, $5 \mathrm{H})$.

${ }^{13} \mathrm{C}$ NMR (50 MHz, $\left.\mathrm{CDCl}_{3}\right) \delta$ 18.0, 21.3 (2C), 71.6, 85.4, 113.8, 123.1, 127.1, 128.1, 128.6, 138.3, 139.1, 157.8. 
<smiles>COc1cc(O)c(C(c2ccccc2)C(O)C(C)=O)c(OC)c1</smiles>

$\left(2 R^{*}, 3 R^{*}\right)$-Methyl 2-hydroxy-3-(2-hydroxy-4,6dimethoxyphenyl)-3-phenylpropanoate (3ca) (table 1, entry 3).

Using the general procedure, a solution of tris-(3,5dimethoxyphenyl) borate (1a) (705 mg, $1.5 \mathrm{mmol}$ ) in $\mathrm{CH}_{2} \mathrm{Cl}_{2}$

$(1.0 \mathrm{~mL})$ was added at $-78^{\circ} \mathrm{C}$ to a solution of trans-methyl-2,3-epoxy-3-phenylpropanoate (2c) (178 mg, $1.0 \mathrm{mmol})$ in $\mathrm{CH}_{2} \mathrm{Cl}_{2}(0.5 \mathrm{~mL})$. The mixture was allowed to react for 18 hours at $-78^{\circ} \mathrm{C}$. The crude mixture, containing $28 \%$ of $\mathrm{O}$-alkylated product, was purified by column chromatography eluting with hexanes/AcOEt 7:3 to give compound 3ca (containing 40\% of $2 R^{*}, 3 S^{*}$-stereoisomer) (182.6 mg, 55\%).

${ }^{1} \mathrm{H}$ NMR (250 MHz, CDCl $) \delta 3.63$ (s, 3H); 3.71 (s, 3H); 3.73 (s, 3H); 5.18 (d, 1H, J= $3.3 \mathrm{~Hz}$ ); 5.35 (d, 1H, J= $3.3 \mathrm{~Hz}$ ); 6.06 (d, 1H, J=2.2 Hz); 6.14 (d, 1H, J=2.2 Hz); 7.13-7.33 (m, 5H).

${ }^{13} \mathrm{C}$ NMR (62.5 MHz, $\left.\mathrm{CDCl}_{3}\right) \delta 41.8,52.6,55.0,55.8,73.2,91.1,95.2,106.0,126.3$, 127.4,128.2,139.6,157.5,158.9,160.6,173.8.

Representative signals of $\left(2 R^{*}, 3 S^{*}\right)$-stereoisomer (anti-3ca):

${ }^{1} \mathrm{H}$ NMR (250 MHz, $\mathrm{CDCl}_{3}$ ) $\delta 4.76$ (d, 1H, J= $\left.2.3 \mathrm{~Hz}, \mathrm{CH}-\mathrm{Ar}\right) ; 5.28$ (d, 1H, J= 2.2 $\mathrm{Hz}, \mathrm{CH}-\mathrm{OH})$.

${ }^{13} \mathrm{C}$ NMR (62.5 MHz, $\left.\mathrm{CDCl}_{3}\right) \delta 43.2(\mathrm{CH}-\mathrm{Ar}), 52.9\left(\mathrm{COOCH}_{3}\right), 73.8(\mathrm{CH}-\mathrm{OH})$.

\section{Clevage of epoxide 2c with borate $1 b$ and directly cyclization of compound 3cb to} the corrisponding 2,3-dihydrobenzofuranes $4 \mathrm{cb}$ and $5 \mathrm{cb}$ (table 1, entry 4).

Using the general procedure, a solution of tris-(3,5-dimethylphenyl) borate (1b) (560.7 mg, $1.5 \mathrm{mmol}$ ) in $\mathrm{CH}_{2} \mathrm{Cl}_{2}(1.0 \mathrm{~mL}$ ) was added at rt under argon to a solution of trans-methyl-2,3-epoxy-3-phenyl-propanoate (2c) (178 mg, $1.0 \mathrm{mmol})$ in $\mathrm{CH}_{2} \mathrm{Cl}_{2}(0.5$ $\mathrm{mL}$ ). The mixture was allowed to react for 18 hours at rt. The reaction was then quenched and the solvent was evaporated under a vacuum. To the crude mixture, containing $50 \%$ of $O$-alkylated product and a 89/11 mixture of syn-3cb and anti-3cb was not isolated but, dissolved in THF $(2.0 \mathrm{~mL})$ and additioned with triphenylphosphine (262.3 mg, $1.0 \mathrm{mmol}$ ) and diethylazodicarboxylate (130.7 mg, $0.118 \mathrm{~mL}, 0.75 \mathrm{mmol})$. The mixture was allowed to react for 2 hours at rt. The purification by flash column chromatography eluting with hexanes/ $\mathrm{Et}_{2} \mathrm{O}$ 9:1 afforded 
(2S*,3R*)-2-carbomethoxy-3-phenyl-4,6-dimethyl-2,3-dihydrobenzofuran

(contaminated with $11 \%$ of $\left(2 R^{*}, 3 R^{*}\right)$ steroisomer $\left.5 \mathbf{c b}\right)$, as a solid (107.2 $\left.\mathrm{mg}, 38 \%\right)$.

${ }^{1} \mathrm{H}$ NMR (250 MHz, CDCl $) \delta 1.88$ (s, 3H); 2.30 (s, 3H); 3.28 (s, 3H); 4.75 (d, 1H, J= $9.5 \mathrm{~Hz}$ ); 5.43 (d, 1H, J=9.5 Hz); 6.53 (s, 1H); 6.67 (s, 1H); 7.00 (d, 1H, J= $7.5 \mathrm{~Hz}$ ); 7.10-7.33 (m, 2H); 7.73 (d, 1H, J= $7.5 \mathrm{~Hz}) .{ }^{13} \mathrm{C}$ NMR (62.5 MHz, $\left.\mathrm{CDCl}_{3}\right) \delta 18.3$, 21.5, 50.7, 51.6, 84.9, 108.3, 124.0, 127.5,128.2, 128.5,129.0, 138.2, 139.4,168.8.

Representative signals of compound 5cb: ${ }^{1} \mathrm{H}$ NMR (250 MHz, $\left.\mathrm{CDCl}_{3}\right) \delta 4.66(\mathrm{~d}, 1 \mathrm{H}$, $J=4.6 \mathrm{~Hz}$ ), 4.97 (d, $1 \mathrm{H}, J=4.6 \mathrm{~Hz}$ ).

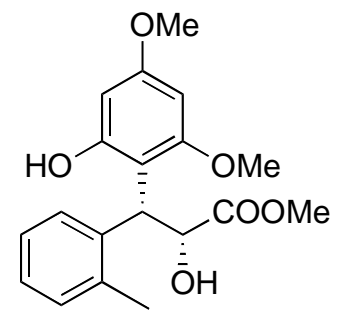

\section{$\left(2 R^{*}, 3 R^{*}\right)$-Methyl 2-hydroxy-3-(2-hydroxy-4,6-}

dimethoxyphenyl)-3-(2-methylphenyl)propanoate (3da) (table 1, entry 5).

Using the general procedure, a solution of tris-(3,5dimethoxyphenyl) borate (1a) (705 mg, $1.5 \mathrm{mmol}$ ) in $\mathrm{CH}_{2} \mathrm{Cl}_{2}$ $(1.0 \mathrm{~mL})$ was added at $-78^{\circ} \mathrm{C}$ to a solution of trans-methyl-3-(2-methylphenyl)-2,3epoxypropanoate (192 mg, $1.0 \mathrm{mmol})$ (2d) in $\mathrm{CH}_{2} \mathrm{Cl}_{2}(0.5 \mathrm{~mL})$. The mixture was allowed to react for 18 hours at $-78^{\circ} \mathrm{C}$. The crude mixture, containing $30 \%$ of $\mathrm{O}$ alkylated product, was purified by column chromatography eluting with hexanes/AcOEt 7:3 to give compound 3da (179.9 mg, 52\%) as a white solid. M.p= $153-155^{\circ} \mathrm{C} . \mathrm{R}_{\mathrm{F}}=0.16$ (hexanes/AcOEt 7:3).

${ }^{1} \mathrm{H}$ NMR (250 MHz, $\mathrm{CDCl}_{3}$ ) $\delta 2.02$ (s, 3H); 3.67 (s, 3H); 3.73 (s, 3H); 3.74 (s, 3H) 4.97-5.04 (m, 1H); 5.15 (d, 1H, J= 2.0 Hz); 5.99 (s, 2H); 7.05-7.24 (m, 3H); 7.69-7.72 (m, 1H).

${ }^{13} \mathrm{C}$ NMR (62.5 MHz, $\left.\mathrm{CDCl}_{3}\right) \delta$ 19.2, 41.0, 52.6, 55.0, 55.6, 73.2, 90.9, 95.2, 104.9, 125.9, 126.7, 127.2, 130.9, 136.6, 139.3, 157.6, 158.7, 160.4, 173.8.

Anal. Calcd. For $\mathrm{C}_{19} \mathrm{H}_{22} \mathrm{O}_{6}$ : C, 65.88\%; H, 6.40\%. Found: C, 65.51\%; H, 6.32\%.<smiles>COc1cc(O)c([C@H](c2ccc(F)cc2)[C@H](O)C(C)=O)c(OC)c1</smiles>

(2R*, 3R*)-Methyl 3-(4-fluorophenyl)-2-hydroxy-3-(2hydroxy-4,6-dimethoxyphenyl)propanoate (3ea) (table 1, entry 6).

Using the general procedure, a solution of tris-(3,5dimethoxyphenyl) borate (1a) (705 $\mathrm{mg}, 1.5 \mathrm{mmol})$ in 
$\mathrm{CH}_{2} \mathrm{Cl}_{2}(1.0 \mathrm{~mL})$ was added at $-40^{\circ} \mathrm{C}$ to a solution of trans-methyl-2,3-epoxy-3-(4fluorophenyl)propanoate (2e) (196 mg, $1.0 \mathrm{mmol})$ in $\mathrm{CH}_{2} \mathrm{Cl}_{2}(0.5 \mathrm{~mL})$. The mixture was allowed to react for 18 hours at $-40^{\circ} \mathrm{C}$. Column chromatography eluting with hexanes/AcOEt 7:3 afforded compound 3ea (217 mg, 54\% yield of 3ea), as a solid, contaminated by an unseparable by-product (ca 15\% of the mixture).

${ }^{1} \mathrm{H}$ NMR (200 MHz, $\mathrm{CDCl}_{3}$ ) $\delta 3.68$ (s, 3H); 3.72, (s, 3H); 3.75 (s, 3H); 4.21-4.34 OH (br, 1H); 5.08-5.18 (m, 1H); 5.25-5.35 (m, 1H); 6.05 (s, 2H); 7.94-7.40 (m, 4H); 8.30 $\mathrm{OH}$ (br, s, 1H).

${ }^{13} \mathrm{C}$ NMR (50 MHz, $\left.\mathrm{CDCl}_{3}\right) \delta 41.2,52.7,55.1,55.8,73.3,91.2,95.2,114.8,115.3$, 127.0, 129.1, 157.4, 159.8, 160.7, 163.8, 173.7.<smiles>COc1cc(O)c(C(c2ccccc2)[C@H](O)CO)c(OC)c1</smiles>

phenylpropanol (2f) (150 mg, $1.0 \mathrm{mmol})$ in $\mathrm{CH}_{2} \mathrm{Cl}_{2}(0.5 \mathrm{~mL})$. The mixture was allowed to react for 18 hours at $-78^{\circ} \mathrm{C}$. Column chromatography eluting with $\mathrm{CH}_{2} \mathrm{Cl}_{2} / \mathrm{AcOEt}$ 7:3 afforded pure 3fa (258.4 mg, 85\%), as a white solid. M.p= 107$109^{\circ} \mathrm{C}$.

${ }^{1} \mathrm{H}$ NMR (250 MHz, MeOD) $\delta 3.37$ (d, 1H, $J=7.7 \mathrm{~Hz}$ ); 3.51-3.58 (m, 1H); 3.62 (s, 3H); 3.64 (s, 3H); 4.53 (d, 1H, J= $9.5 \mathrm{~Hz}) ; 4.81-4.97$ (m, 1H); 5.97 (s,1H); 6.02 (s,1H); 7.01-7.08 (m, 1H); 7.16 (t, 2H, $J=7 . .5 \mathrm{~Hz}) ; 7.47$ (d, 2H, $J=7.5 \mathrm{~Hz})$.

${ }^{13} \mathrm{C}$ NMR (62.5 MHz, MeOD) $\delta$ 45.3, 55.5, 55.6, 66.6, 73.7, 91.5, 95.0, 111.4, 126.4, 128.5, 130.1, 144.4, 157.4, 160.3, 161.0.

Anal. Calcd. For $\mathrm{C}_{17} \mathrm{H}_{20} \mathrm{O}_{5}$ : C, 67.09\%; H, 6.62\%. Found: C, 67.32\%; H, 6.50\%.

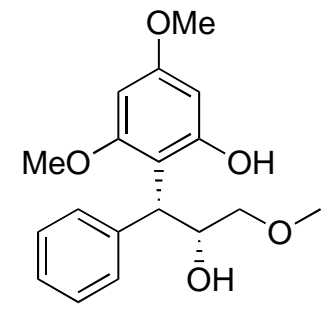

2-((1R*,2R*)-2-Hydroxy-3-methoxy-1-phenylpropyl)-3,5dimethoxyphenol (Syn-3ga) (table 1, entry 8).

Using the general procedure, a solution of tris (3,5dimethoxyphenyl) borate (1a) (705 $\mathrm{mg}, 1.5 \mathrm{mmol}$ ) in $\mathrm{CH}_{2} \mathrm{Cl}_{2}$ $(1.0 \mathrm{~mL})$ was added at $-78^{\circ} \mathrm{C}$ to a solution of trans-2(methoxymethyl)-3-phenyloxirane (2g) (164 mg, $1.0 \mathrm{mmol})$ in $\mathrm{CH}_{2} \mathrm{Cl}_{2}(0.5 \mathrm{~mL})$. The 
mixture was allowed to react for 18 hours at $-78^{\circ} \mathrm{C}$. The crude mixture, containing $23 \%$ of $O$-alkylated product, was purified by column chromatography eluting with hexanes/AcOEt 7:3 to give compound syn-3ga (contaminated with $5 \%$ of $O$-alkylated product, see below) (165.4 mg, 52\%), as a white solid.

${ }^{1} \mathrm{H}$ NMR (250 MHz, $\mathrm{CDCl}_{3}$ ) $\delta$ 3.15-3.27 (m, 4H); 3.46 (dd, 1H, $J_{1}=9.7 \mathrm{~Hz}, J_{2}=2.5$ $\mathrm{Hz}) ; 3.70$ (s, 3H); 3.77 (s, 3H); $4.24 \mathrm{CHOH}$ (br, s, 1H); 4.71-4.80 (m, 1H); 4.88 (d, $1 \mathrm{H}, J=2.7 \mathrm{~Hz}$ ); 6.07 (d, 1H, $J=2.2 \mathrm{~Hz}) ; 6.17$ (d, 1H, $J=2.2 \mathrm{~Hz}) ; 7.11-7.40$ (m, 5H); 9.40 $\mathrm{ArOH}(\mathrm{br}, \mathrm{s}, 1 \mathrm{H})$.

${ }^{13} \mathrm{C}$ NMR (62.5 MHz, $\left.\mathrm{CDCl}_{3}\right) \delta 40.8,55.1,55.8,58.9,72.9,75.4,90.9,95.0,106.4$, 126.1, 127.8, 128.2, 140.4, 157.2, 159.1, 160.2.

Representative signals of the $O$-alkylated product:

${ }^{1} \mathrm{H}$ NMR (250 MHz, $\left.\mathrm{CDCl}_{3}\right) \delta$ 3.93-4.08 (m, 1H); 5.21 (d, 1H, J=6.75 Hz)

${ }^{13} \mathrm{C}$ NMR (62.5 MHz, $\left.\mathrm{CDCl}_{3}\right) \delta$ 59.1 $\left(\mathrm{CH}_{2} \mathrm{OCH}_{3}\right), 74.4(\mathrm{CHOH}), 80.6$ (CHOAr).<smiles>COC[C@H](O)C(c1ccccc1)c1c(O)cc(OC)cc1OC</smiles>
2-((1S*,2R*)-2-Hydroxy-3-methoxy-1-phenylpropyl)-3,5dimethoxyphenol (anti-3ga) (table 1, entry 8). The first eluting fractions of the above described column chromatography afforded pure 2-((1S*,2R*)-2-hydroxy-3methoxy-1-phenylpropyl)-3,5-dimethoxyphenol

(anti-3ga) (contamined with $4 \%$ of $O$-alkylated product) (yield=14\%), as an oil.

${ }^{1} \mathrm{H}$ NMR (250 MHz, $\mathrm{CDCl}_{3}$ ) $\delta$ 3.28-3.45 (m, 4H); 3.51 (dd, 1H, $J_{1}=9.75 \mathrm{~Hz}, J_{2}=3.75$ $\mathrm{Hz}$ ); 4.50-4.60 (m, 1H); 4.64 (d, 1H, $J=4.0 \mathrm{~Hz}$ ); 5.97 (d, 1H, $J=2.25 \mathrm{~Hz}$ ); 6.11 (d, $1 \mathrm{H}, J=2.25 \mathrm{~Hz}$ ); 7.08-7.41 (m, 5H); 9.09 $\mathrm{ArOH}$ (br, s, 1H).

${ }^{13} \mathrm{C} \mathrm{NMR}\left(62.5 \mathrm{MHz}, \mathrm{CDCl}_{3}\right) \delta 42.5,55.1,56.6,58.9,71.7,75.2,91.2,94.8,110.2$, 126.3, 127.7, 129.5, 156.4, 158.6, 159.8 .<smiles>COc1cc(O)c([C@H](c2ccccc2)[C@H](O)C(C)=O)c(OC)c1</smiles>

$\left(2 R^{*}, 3 R^{*}\right)$-Methyl 2-hydroxy-3-(2-hydroxy-4,6dimethoxyphenyl)-3-phenylpropanoate (3ca) (table 1, entry 10).

Using the general procedure, a solution of tris (3,5dimethoxyphenyl) borate (1a) (705 mg, $1.5 \mathrm{mmol}$ ) in $\mathrm{CH}_{2} \mathrm{Cl}_{2}$ 
$(1.0 \mathrm{~mL})$ was added at $-78^{\circ} \mathrm{C}$ to a solution of cis-methyl-2,3-epoxy-3-phenylpropanoate ${ }^{1}$ (2i) $(178 \mathrm{mg}, 1.0 \mathrm{mmol})$ in $\mathrm{CH}_{2} \mathrm{Cl}_{2}(0.5 \mathrm{~mL})$. The mixture was allowed to react for 18 hours at $-78^{\circ} \mathrm{C}$. The crude mixture, containing $12 \%$ of $\mathrm{O}$-alkylated product, was purified by column chromatography eluting with hexanes/AcOEt 7:3 to give compound 3ca (containing 13\% of trans-stereoisomer) (172.6 mg, 52\%).

For ${ }^{1} \mathrm{H}$ NMR and ${ }^{13} \mathrm{C}$ NMR data see above.

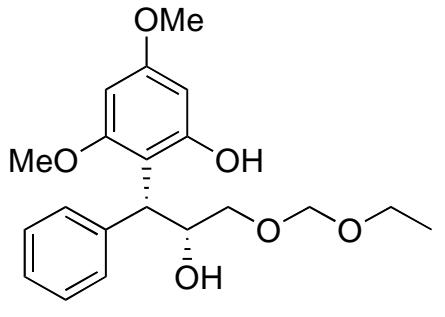

2-((1R*,2R*)-3-(Ethoxymethoxy)-2-hydroxy-1phenyl propyl)-3,5-dimethoxyphenol (3ja) (table 1, entry 11).

Using the general procedure, a solution of tris $(3,5-$ dimethoxyphenyl) borate (1a) (705 $\mathrm{mg}, 1.5 \mathrm{mmol}$ ) in $\mathrm{CH}_{2} \mathrm{Cl}_{2}(1.0 \mathrm{~mL})$ was added at $-78^{\circ} \mathrm{C}$ to a solution of cis-2-((ethoxymethoxy)methyl)3-phenyloxirane $2 \mathbf{j}$ (208 mg, $1.0 \mathrm{mmol}$ ) in $\mathrm{CH}_{2} \mathrm{Cl}_{2}(0.5 \mathrm{~mL})$. The mixture was allowed to react for 18 hours at $-78^{\circ} \mathrm{C}$. Column chromatography eluting with hexanes/AcOEt 7:3 afforded pure 3ja (253.4 mg, 70\%), as an oil. $\mathrm{R}_{\mathrm{F}}=0.16$ (hexanes/AcOEt 7:3).

${ }^{1} \mathrm{H}$ NMR (250 MHZ, CDCl 3 ) $\delta 1.20\left(\mathrm{t}, 3 \mathrm{H}, J=7.1 \mathrm{~Hz}\right.$ ); $3.32\left(\mathrm{dd}, 1 \mathrm{H}, J_{1}=11.5 \mathrm{~Hz}, J_{2}=\right.$ $9.3 \mathrm{~Hz}$ ) 3.50-3.73 (m, 3H); 3.71 (s, 3H); 3.76 (s, 3H); 3.83 (dd, $1 \mathrm{H}, J_{1}=11.2 \mathrm{~Hz}, J_{2}=$ $2.4 \mathrm{~Hz}$ ); 4.54-4.80 (m, 3H); 4.91 (d, 1H, J= $3.1 \mathrm{~Hz}$ ); 6.07 (d, 1H, J= 2.4 Hz); 6.15 (d, $1 \mathrm{H}, J=2.4 \mathrm{~Hz}$ ); 7.11-7.39 (m, 5H); $9.42 \mathrm{ArOH}$ (br, s, 1H).

${ }^{13} \mathrm{C}$ NMR $\left(62.5 \mathrm{MHz}, \mathrm{CDCl}_{3}\right) \delta 15.0,40.8,55.1,55.8,63.8,73.4,73.5,91.0,95.2$, 96.1, 106.5, 126.2, 127.8, 128.3, 140.6, 157.3, 159.2, 160.4.

Anal. Calcd. For $\mathrm{C}_{20} \mathrm{H}_{26} \mathrm{O}_{6}$ : C, 66.28\%; H, 7.23\%. Found: C, 66.30\%; H, 7.23\%.

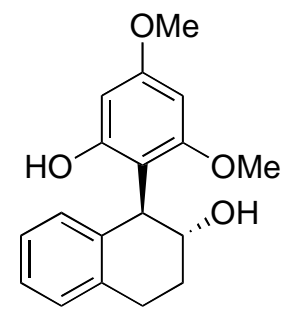

$\left(1 S^{*}, 2 R^{*}\right)-1-(2-H y d r o x y-4,6-d i m e t h o x y p h e n y l)-1,2,3,4-t e t r a$ hydronaphtalen-2-ol (3ka) (table 1, entry 12).

Using the general procedure, a solution of tris-(3,5dimethoxyphenyl) borate (1a) (705 mg, $1.5 \mathrm{mmol})$ in $\mathrm{CH}_{2} \mathrm{Cl}_{2}(1.0$ $\mathrm{mL}$ ) was added at $-80^{\circ} \mathrm{C}$ to a solution of dihydronaphtalene oxide (2k) (146 mg, $1.0 \mathrm{mmol})$ in $\mathrm{CH}_{2} \mathrm{Cl}_{2}(0.5 \mathrm{~mL})$. The mixture was allowed to react for 18 hours at $-78^{\circ} \mathrm{C}$. Column chromatography eluting with hexanes/AcOEt 6:4 afforded pure 3ka (156 mg, 52\%), as a solid. M.p= $150-152{ }^{\circ} \mathrm{C}$.

\footnotetext{
${ }^{1}$ Denis, J.N; Greene, A. E; Serra, A. A; Luche M. J. J. Org. Chem. 1986, 51, 46-50.
} 
${ }^{1} \mathrm{H}$ NMR (250 MHz, $\left.\mathrm{CDCl}_{3}\right) \delta$ 1.75-1.92 (m,1H); 2.16-2.26 (m, 1H); 2.84-3.08 (m, 2H); 3.60 (br, s, 3H); 3.72 (s, 3H); 4.19 (ddd, 1H, $J_{1}=11.0 \mathrm{~Hz}, J_{2}=9.0 \mathrm{~Hz}, J_{3}=3.5$ $\mathrm{Hz}$ ); 4.47 (d, 1H, $J=9.0 \mathrm{~Hz}$ ); 6.03 (d, 1H, $J=2.3 \mathrm{~Hz}) ; 6.10$ (d,1H, $J=2.3 \mathrm{~Hz}) ; 6.85$ (d, $1 \mathrm{H}, J=7.2 \mathrm{~Hz}) ; 6.93-7.10(\mathrm{~m}, 3 \mathrm{H})$.

${ }^{13} \mathrm{C}$ NMR (62.5 MHz, $\left.\mathrm{CDCl}_{3}\right) \delta$ 28.6, 31.6, 44.0, 55.2, 55.7, 71.9, 92.0, 94.9, 126.3, 127.8,128.4,136.0,157.1, 159.8, 160.1.

Anal. Calcd. For $\mathrm{C}_{18} \mathrm{H}_{20} \mathrm{O}_{4}$ : C, 71.98\%; H, 6.71\%. Found: C, 72.03\%; H, 6.44\%.<smiles>Cc1cc(C)c(C(CO)c2ccc(F)cc2)c(O)c1</smiles>

2-(1-(4-Fluorophenyl)-2-hydroxyethyl)-3,5-dimethylphenol (3mb) (substrate of table 2, entry 5)

Using the general procedure, a solution of tris $(3,5-$ dimethylphenyl) borate (1b) (560.7 mg, $1.5 \mathrm{mmol}$ ) in $\mathrm{CH}_{2} \mathrm{Cl}_{2}$ $(1.0 \mathrm{~mL})$ was added at $-78^{\circ} \mathrm{C}$ under argon to a solution of $p$ fluorophenyl oxirane (2m) (138.1 $\mathrm{mg}, 1.0 \mathrm{mmol})$ in $\mathrm{CH}_{2} \mathrm{Cl}_{2}(0.5 \mathrm{~mL})$. The mixture was allowed to react for 1.5 hours at $-78^{\circ} \mathrm{C}$. The crude mixture (containing $16 \%$ of $O-$ alkylated product) was purified by column chromatography eluting with hexanes/AcOEt 7:3 to give compound 3mb (150.8 mg, 58\%), as a white solid. M.p= 119- $121^{\circ} \mathrm{C} . \mathrm{R}_{\mathrm{f}}=0.25$ (hexanes/AcOEt 7:3).

${ }^{1} \mathrm{H}$ NMR (200 MHz, MeOD) $\delta$ 2.13-2.22 (m, 6H); 4.16-4.49 (m, 3H); 6.43-6.53 (m, 2H); 6.83-6.98 (m, 2H); 7.24-7.39 (m, 2H).

${ }^{13} \mathrm{C}$ NMR (50 MHz, MeOD) $\delta 20.3$ (2 C), 47.1, 65.2, 114.4, 114.8, 115.2, 123.3, 124.5, 130.1, 130.3, 137.4, 138.3, 138.9, 156.0, 159.6, 164.2.

Anal. Calcd. For $\mathrm{C}_{16} \mathrm{H}_{17} \mathrm{FO}_{2}$ : C, 73.83\%; H, 6.58\%. Found: C, 73.53\%; H, 6.48\%.

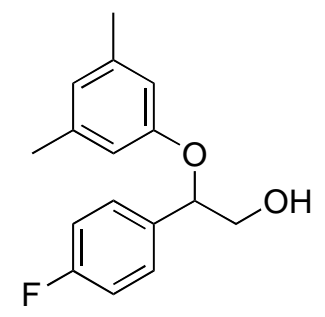

\section{2-(3,5-Dimethylphenoxy)-2-(4-fluorophenyl)-ethanol.}

The first eluting fractions of the above described column chromatography $\left(\mathrm{R}_{\mathrm{f}}=0.35\right.$ in hexanes/AcOEt 7:3) afforded pure

2-(3,5-dimethylphenoxy)-2-(4-fluorophenyl)-ethanol as a semisolid. (19.8 mg, 7.6\%).

${ }^{1} \mathrm{H}$ NMR (200 MHz, $\mathrm{CDCl}_{3}$ ) $\delta 2.22$ (s, 6H); 3.68-3.95 (m, 2H); 5.24 (dd, 1H, $J_{1}=7.8$ $\mathrm{Hz}, J_{2}=4.0 \mathrm{~Hz}$ ); 6.45-6.60 (m, 3H); 6.97-7.10 (m, 2H); 7.25-7.40 (m, 2H).

${ }^{13} \mathrm{C}$ NMR (50 MHz, $\left.\mathrm{CDCl}_{3}\right) \delta 21.4$ (2 C), 67.5, 80.2, 113.7, 115.5, 115.9, 123.2, 127.8, 128.0, 133.8, 139.2, 157.6, 164.9. 
COH

2-(2-Hydroxy-1-(4-methylphenyl)-ethyl)-3,5-dimethyl phenol ${ }^{2}$ (3nb) (substrate of table 2, entry 6).

Using the general procedure, a solution of tris-(3,5dimethylphenyl) borate (1b) (560.7 $\mathrm{mg}, 1.5 \mathrm{mmol})$ in $\mathrm{CH}_{2} \mathrm{Cl}_{2}$ $(1.0 \mathrm{~mL})$ was added at $-78^{\circ} \mathrm{C}$ under argon to a solution of $p$ methylphenyl oxirane (2n) (134.1 mg, $1.0 \mathrm{mmol})$ in $\mathrm{CH}_{2} \mathrm{Cl}_{2}(0.5 \mathrm{~mL})$. The mixture was allowed to react for 1.5 hours at $-78^{\circ} \mathrm{C}$. The crude mixture (containing $13 \%$ of $O$ alkylated product) was purified by column chromatography eluting with hexanes/AcOEt 8:2 to give compound 3nb (143.4 mg, 56\%), as a liquid. $\mathrm{R}_{\mathrm{f}}=0.15$ (hexanes/AcOEt 8:2).

${ }^{1} \mathrm{H}$ NMR (200 MHz, $\left.\mathrm{CDCl}_{3}\right) \delta 2.25$ (s, 3H); 2.31 (s, 3H); 2.36 (s, 3H); 2.96-3.10 CHOH (br, 1H); 4.17-4.28 (m, 1H); 4.37-4.55 (m, 2H); 6.62-6.70 (m, 2H); 7.09-7.32 (m, 4H); 8.36-8.48 $\mathrm{ArOH}$ (m, 1H).

${ }^{13} \mathrm{C}$ NMR (50 MHz, $\left.\mathrm{CDCl}_{3}\right) \delta 20.8$ (3 C), 45.9, 65.1, 116.4, 123.0, 123.5, 127.8, $129.5,136.2,136.4,137.7,138.1,155.2$.

Anal. Calcd. For $\mathrm{C}_{17} \mathrm{H}_{20} \mathrm{O}_{2}$ : C, 79.65\%; H, 7.86\%. Found: C, 79.74\%; H, 7.63\%.<smiles>OCC(c1ccccc1)c1c(O)ccc2ccccc12</smiles>

\section{1-((1S)-2-Hydroxy-1-phenylethyl)naphtalen-2-ol (3ld) (substrate} of table 2, entry 7).

Using the general procedure, a solution of tris ( $\beta$-naphtyl) borate (1d) $(660 \mathrm{mg}, 1.5 \mathrm{mmol})$ in $\mathrm{CH}_{2} \mathrm{Cl}_{2}(1.0 \mathrm{~mL})$ was added at $-78^{\circ} \mathrm{C}$ under argon to a solution of $(R)-(+)$-styrene oxide (2l) $(120.1 \mathrm{mg}, 1.0 \mathrm{mmol})$ in $\mathrm{CH}_{2} \mathrm{Cl}_{2}(0.5 \mathrm{~mL})$. The mixture was allowed to react for 1.5 hours $-78^{\circ} \mathrm{C}$. The crude mixture (containing $17 \%$ of $O$-alkylated product) was purified by column chromatography eluting with hexanes/AcOEt 8:2 to give compound 3ld (179.5 mg, 68 \%) (containing $24 \%$ of $(R)$-stereoisomer) as a white solid. M.p $=112-114^{\circ} \mathrm{C} . \mathrm{R}_{\mathrm{f}}=0.26$ (hexanes/AcOEt 7:3). $[\alpha]_{\mathrm{D}}{ }^{20}=-49.6\left(\mathrm{c}=1.0, \mathrm{CHCl}_{3}\right)$.

${ }^{1} \mathrm{H}$ NMR (250 MHz, $\mathrm{CDCl}_{3}$ ) $\delta$ 2.75-3.20 OH (br, 1H); 4.32 (m, 1H); 4.47 (dd, $1 \mathrm{H}, J_{1}=$ $\left.11.4 \mathrm{~Hz}, J_{2}=4.9 \mathrm{~Hz}\right)$; 5.05-5.20 (m, 1H); 7.12-7.50 (m, 8H); 7.68-7.90 (m, 3H); $9.00-$ 9.35 $\mathrm{ArOH}(\mathrm{br}, 1 \mathrm{H})$. 
${ }^{13} \mathrm{C}$ NMR $\left(62.5 \mathrm{MHz}, \mathrm{CDCl}_{3}\right) \delta 45.8,65.4,117.5,120.3,122.3,122.8,126.7,126.9$, 128.2, 128.8, 129.0, 129.3, 129.5, 133.7, 139.8, 153.4 .

Anal. Calcd. For $\mathrm{C}_{18} \mathrm{H}_{16} \mathrm{O}_{2}$ : C, 81.79\%; H, 6.10\%. Found: C, 81.55\%; H, 6.06\%.

HPLC analysis performed on a Daicel Chiralcel ${ }^{\circledR}$ OD-H column, flow rate: 0.5 $\mathrm{mL} / \mathrm{min}$, mobile phase: hexane/isopropanol 90/10, retention times (min): 27.3 ( $R$, minor stereoisomer), 39.6 ( $S$, major stereoisomer).

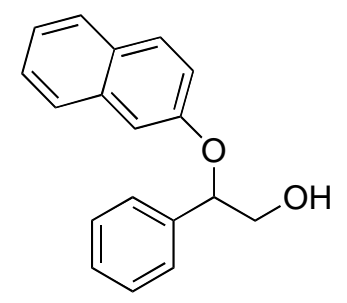

\section{2-(Naphtalen-2-yloxy)-2-phenylethanol.}

The first eluting fractions of the above described column chromatography $\left(\mathrm{R}_{\mathrm{f}}=0.35\right.$ in hexanes/AcOEt 7:3) afforded pure 2-naphtoxy-2-phenyl-ethanol as a semisolid. (15.8 mg, $6.6 \%)$.

${ }^{1} \mathrm{H}$ NMR (250 MHz, $\left.\mathrm{CDCl}_{3}\right) \delta$ 2.68- $2.88 \mathrm{OH}(\mathrm{br}, 1 \mathrm{H}) ; 3.80-4.06$ (m, 2H); 5.41 (dd, $\left.1 \mathrm{H}, J_{1}=8.0 \mathrm{~Hz}, J_{2}=3.7 \mathrm{~Hz}\right) ; 7.01-7.50(\mathrm{~m}, 10 \mathrm{H}) ; 7.56$ (d, $\left.1 \mathrm{H}, J=7.9 \mathrm{~Hz}\right) ; 7.62-7.80$ (m, 2H).

\section{Synthesis of 2,3-dihydrobenzofuranes by Mitsunobu cyclodehydration (Table 2).}

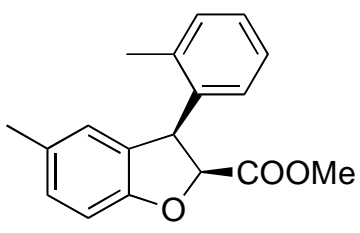

(2S*,3R*)-Methyl 5-methyl-3-(2-methylphenyl)-2,3dihydrobenzofuran-2-carboxylate(4dc) (table 2, entry 1). Using the general procedure described in the main text, to a solution of $\left(2 R^{*}, 3 R^{*}\right)$-methyl-2-hydroxy-3-(2-hydroxy-5methylphenyl)-3-(2-methylphenyl)propanoate ${ }^{2}$ (3dc) $(60.0 \mathrm{mg}, \quad 0.2 \mathrm{mmol})$ in anhydrous THF (0.8 mL) were added triphenylphosphine (104.9 mg, $0.4 \mathrm{mmol})$ and diethylazodicarboxylate (52.2 mg, $0.3 \mathrm{mmol})$. The mixture was allowed to react for 4 hours at rt. Column chromatography eluting with hexanes/Et $\mathrm{E}_{2} \mathrm{O}$ 9:1 afforded pure 4dc (35.0 mg, 62\%), as a solid. M.p $=105-107^{\circ} \mathrm{C}$.

${ }^{1} \mathrm{H}$ NMR (200 MHz, $\mathrm{CDCl}_{3}$ ) $\delta 2.26$ (s, 3H); 2.48 (s, 3H); 3.19 (s, 3H); 5.25 (d, 1H, $J=10.2$ Hz); 5.50 (d, 1H, $J=10.2$ Hz); 6.81-6.96 (m, 3H); 6.99-7.22 (m, 4H).

${ }^{13} \mathrm{C}$ NMR (50 MHz, $\left.\mathrm{CDCl}_{3}\right) \delta$ 19.3, 20.8, 47.3, 51.4, 83.7, 109.6, 125.8, 126.1, 127.4, 128.4, 129.3, 129.8, 131.0, 137.0, 174.1 .

\footnotetext{
2 Bertolini, F.; Crotti, P.; Macchia, F.; Pineschi, M. Tetrahedron Lett. 2006, 47, 61.
} 
Anal. Calcd. For $\mathrm{C}_{18} \mathrm{H}_{18} \mathrm{O}_{3}$ : C, 76.57\%; H, 6.43\%. Found: C, 76.49\%; H, 6.29\%.

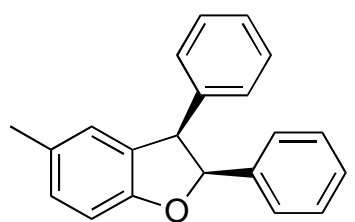

$\left(2 S^{*}, 3 R^{*}\right)$-5-Methyl-2,3-diphenyl-2,3-dihydrobenzofuran (4bc) (table 2, entry 2).

Using the general procedure, to a solution of $\left(2-\left(\left(1 R^{*}, 2 R^{*}\right)-\right.\right.$ 1,2-diphenylethyl-2-hydroxy)-4-methylphenol ${ }^{2} \quad$ (3bc) (45.6 mg, $0.15 \mathrm{mmol})$ in anhydrous THF $(0.6 \mathrm{~mL})$ were added triphenylphosphine (78.8 $\mathrm{mg}, 0.3 \mathrm{mmol}$ ) and diethylazodicarboxylate (39.1 $\mathrm{mg}, 0.225 \mathrm{mmol})$. The mixture was allowed to react for 5 hours at rt. Column chromatography eluting with hexanes/Et $2 \mathrm{O}$ 8:2 afforded pure $\mathbf{4 b c}(27.9 \mathrm{mg}, 65 \%)$, as a solid. $\mathrm{M} . \mathrm{p}=70-73^{\circ} \mathrm{C}$.

${ }^{1} \mathrm{H}$ NMR (200 MHz, $\left.\mathrm{CDCl}_{3}\right) \delta 2.28$ (s, 3H); 4.82 (d, 1H, J=8.9 Hz); 6.00 (d, 1H, J= 8.9 Hz); 6.66- 6.73 (m, 2H); 6.91-7.09 (m, 10H); 7.26 (s, 1H).

${ }^{13} \mathrm{C}$ NMR (50 MHz, $\left.\mathrm{CDCl}_{3}\right) \delta$ 20.8, 54.0, 88.7, 109.3, 126.5, 127.2, 127.6, 127.7, 129.2, 130.5, 130.7, 137.6, 139.2.

Anal. Calcd. For $\mathrm{C}_{18} \mathrm{H}_{18} \mathrm{O}_{3}$ : C, 88.08\%; H, 6.34\%. Found: C, 88.11\%; H, 6.12\%.

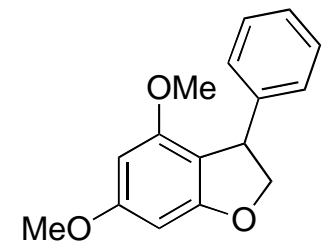

4,6-Dimethoxy-3-phenyl-2,3-dihydrobenzofuran (4la) (table 2, entry 3).

Using the general procedure, to a solution of 2-(2-hydroxy-1phenylethyl)-3,5-dimethoxyphenol $^{2}$ (3la) (115.1 mg, 0.42 mmol) in anhydrous THF (1.7 mL) were added triphenylphosphine (220.3 mg, 0.84 mmol) and diethylazodicarboxylate (109.6 $\mathrm{mg}, 0.63 \mathrm{mmol})$. The mixture was allowed to react for 2 hours at rt. Column chromatography eluting with hexanes/ $\operatorname{Et}_{2} \mathrm{O}$ 9:1 afforded pure 4 la (102.1 mg, 95\%), as a solid. M.p $=103-105^{\circ} \mathrm{C}$.

${ }^{1} \mathrm{H}$ NMR (200 MHz, $\mathrm{CDCl}_{3}$ ) $\delta 3.65$ (s, 3H); 3.82 (s, 3H); 4.49 (dd, 1H, $J_{1}=8.3 \mathrm{~Hz}$, $\left.J_{2}=4.2 \mathrm{~Hz}\right) ; 4.61\left(\mathrm{dd}, 1 \mathrm{H}, J_{1}=8.9 \mathrm{~Hz}, J_{2}=4.2 \mathrm{~Hz}\right) ; 4.89(\mathrm{t}, 1 \mathrm{H}, J=8.7 \mathrm{~Hz}) ; 6.05(\mathrm{~d}$, $1 \mathrm{H}, J=1.8 \mathrm{~Hz}) ; 6.19$ (d, 1H, $J=1.8 \mathrm{~Hz})$; 7.15-7.38 (m, 5H).

${ }^{13} \mathrm{C}$ NMR (50 MHz, $\left.\mathrm{CDCl}_{3}\right) \delta 45.8,55.2,55.4,80.3,88.3,91.5,109.4,126.5,127.0$, 128.4, 143.5, 157.0, 162.1.

Anal. Calcd. For $\mathrm{C}_{18} \mathrm{H}_{18} \mathrm{O}_{3}$ : C, 74.98\%; H, 6.29\%. Found: C, 75.11\%; H, 6.22\%. 


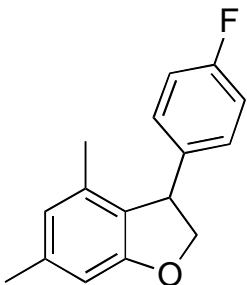

4,6-Dimethyl-3-(4-fluorophenyl)-2,3-dihydrobenzofuran (4mb) (table 2, entry 5).

Using the general procedure, to a solution of 2-(2-hydroxy-1-(4fluorophenyl)-ethyl)-3,5-dimethylphenol (3mb) (109.2 mg, 0.42 mmol) in anhydrous THF (1.7 mL) were added triphenylphosphine (220.3 $\mathrm{mg}, 0.84 \mathrm{mmol}$ ) and diethylazodicarboxylate (109.6 mg, $0.63 \mathrm{mmol})$. The mixture was allowed to react for 2 hours at rt. Column chromatography eluting with hexanes/Et ${ }_{2} \mathrm{O}$ 9:1 afforded pure $\mathbf{4 m b}(89.4 \mathrm{mg}, 88 \%)$, as a solid. M.p= 63- $65^{\circ} \mathrm{C}$.

${ }^{1} \mathrm{H}$ NMR (200 MHz, $\mathrm{CDCl}_{3}$ ) $\delta 1.94$ (s, 3H); 2.33 (s, 3H); 4.39 (dd, 1H, $J_{1}=8.4 \mathrm{~Hz}$, $J_{2}=4.8 \mathrm{~Hz}$ ); 4.49- 4.55 (m, 1H); 4.85 (t, 1H, J= 8.8 Hz); 6.52 (br, s, 1H); 6.60 (br, s, 1H); 6.94-7.15 (m, 4H).

${ }^{13} \mathrm{C}$ NMR $\left(50 \mathrm{MHz}, \mathrm{CDCl}_{3}\right) \delta 18.5,21.4,46.8,79.5,107.9,115.3,115.7,123.2$, 128.8, 129.0, 134.9, 139.1, 159.2, 164,1.

Anal. Calcd. For $\mathrm{C}_{16} \mathrm{H}_{15} \mathrm{FO}$ : C, 79.32\%; H, 6.24\%. Found: C, 79.11\%; H, 6.32\%.

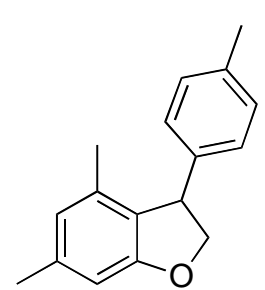

4,6-Dimethyl-3-(4-methylphenyl)-2,3-dihydrobenzofuran (4nb) (table 2, entry 6).

Using the general procedure, to a solution of 2-(2-hydroxy-1-(4methylphenyl)-ethyl)-3,5-dimethylphenol (3nb) (61.4 mg, 0.24 $\mathrm{mmol})$ in anhydrous THF (0.96 $\mathrm{mL})$ were added triphenylphosphine (125.9 mg, $0.48 \mathrm{mmol}$ ) and diethylazodicarboxylate (62.6 mg, $0.36 \mathrm{mmol})$. The mixture was allowed to react for 2 hours at rt. Column chromatography eluting with hexanes/Et ${ }_{2} \mathrm{O}$ 9:1 afforded pure 4nb (48.6 mg, 85\%), as an oil.

${ }^{1} \mathrm{H}$ NMR (200 MHZ, $\mathrm{CDCl}_{3}$ ) $\delta 1.94$ (s, 3H); 2.32 (s, 3H); 2.34 (s, 3H); 4.40 (dd, 1H, $\left.J_{1}=8.2 \mathrm{~Hz}, J_{2}=5.0 \mathrm{~Hz}\right) ; 4.51\left(\mathrm{dd}, 1 \mathrm{H}, J_{1}=8.9 \mathrm{~Hz}, J_{2}=5.0 \mathrm{~Hz}\right) ; 4.85(\mathrm{t}, 1 \mathrm{H}, J=8.5$ Hz); 6.51 (br, s, 1H); 6.59 (br, s, 1H); 7.00-7.15 (m, 4H).

${ }^{13} \mathrm{C}$ NMR (50 MHz, $\mathrm{CDCl}_{3}$ ) $\delta$ 18.5, 21.0, 21.4, 47.2, 79.7, 106.1, 107.7, 110.1, 123.0, 126.0, 127.4, 129.3, 135.1, 136.2, 138.8, 140.3, 160.3.

MS (relative intensity) 238 (M+100), 223 (53), 208 (17), 195 (18), 165 (10), 119 (11). Anal. Calcd. For $\mathrm{C}_{17} \mathrm{H}_{18} \mathrm{O}$ : C, 85.67\%; H, 7.61\%. Found: C, 85.11\%; H, 7.32\%. 


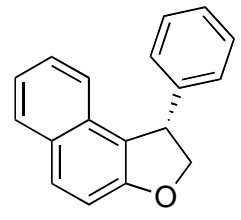

(S)-1-Phenyl-1,2-dihydronaphtho[2,1-b]furan (4ld) (table 2, entry 7).

Using the general procedure, to a solution of 1-((1S)-2-hydroxy-1phenylethyl)naphtalen-2-ol (3ld) ${ }^{3} \quad(127.7 \mathrm{mg}, 0.48 \mathrm{mmol})$ in anhydrous THF (1.92 mL) were added triphenylphosphine (251.8 mg, $0.96 \mathrm{mmol})$ and diethylazodicarboxylate (125.3 mg, $0.72 \mathrm{mmol})$. The mixture was allowed to react for 3 hours at rt. The product (containing $22 \%$ of $(R)$-stereoisomer) was isolated (112.2 mg, 95\%) by column chromatography eluting with hexanes/AcOEt 8:2, as a light brown solid. M.p $=89-90^{\circ} \mathrm{C} .[\alpha]_{\mathrm{D}}{ }^{20}=+45.2\left(\mathrm{c}=3.7, \mathrm{CHCl}_{3}\right)$.

${ }^{1} \mathrm{H}$ NMR $\left(250 \mathrm{MHz}, \mathrm{CDCl}_{3}\right) \delta 4.60\left(\mathrm{dd}, 1 \mathrm{H}, J_{1}=7.8 \mathrm{~Hz}, J_{2}=4.4 \mathrm{~Hz}\right) ; 5.01-5.26(\mathrm{~m}$, 2H); 7.18-7.45 (m, 9H); 7.72-7.90 (m, 2H).

${ }^{13} \mathrm{C}$ NMR $\left(62.5 \mathrm{MHz}, \mathrm{CDCl}_{3}\right) \delta 48.2,80.3,112.1,121.0,122.8,126.6,126.9,127.6$, 128.7, 128.8, 129.7, 130.0, 130.6, 143.3, 158.2.

Anal. Calcd. For $\mathrm{C}_{19} \mathrm{H}_{14} \mathrm{O}$ : C, 87.78\%; H, 5.73\%. Found: C, 87.90\%; H, 5.65\%.

HPLC analysis performed on a Daicel Chiralcel ${ }^{\circledR}$ OD-H column, flow rate: 0.5 $\mathrm{mL} / \mathrm{min}$, mobile phase: hexane/isopropanol 98/2, retention times (min): 10.8 ( $R$, minor stereoisomer), 15.7 ( $S$, major stereoisomer).

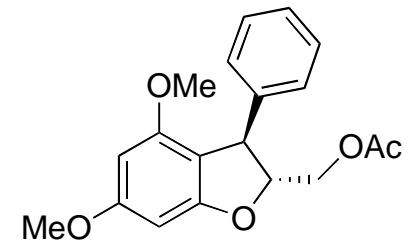

(2 $\left.R^{*}, 3 R^{*}\right)-4,6-D i m e t h o x y-3-p h e n y l-2,3-d i h y d r o b e n z o$ furan-2-yl)methyl acetate (5fa) (table 2, entry 8).

Using the general procedure, to a solution of $\left(2 R^{*}, 3 S^{*}\right)-3-$ (2-hydroxy-4,6-dimethoxy)-3-phenylpropane-1,2-diol (3fa) (100.3 mg, $0.33 \mathrm{mmol})$ in anhydrous THF $(1.32 \mathrm{~mL})$ were added triphenylphosphine $(173.1 \mathrm{mg}, 0.66 \mathrm{mmol})$ and diethylazodicarboxylate $(87.0 \mathrm{mg}$, $0.50 \mathrm{mmol}$ ). The mixture was allowed to react for 5 hours at $\mathrm{rt}$ and then the solvent was evaporated under a vacuum. To the crude mixture dissolved in pyridine $(2.0 \mathrm{~mL})$ was then added at $0^{\circ} \mathrm{C}$ acetic anhydride (673.2 $\left.\mathrm{mg}, 0.62 \mathrm{~mL}, 6.6 \mathrm{mmol}\right)$. The mixture was allowed to react for 24 hours at rt. The mixture was then quenched with aqueous $\mathrm{NaHCO}_{3}$ saturated solution. The organic phase was then washed with $\mathrm{HCl} 10 \%$ and brine, dried over $\mathrm{MgSO}_{4}$ and filtered. Column chromatography eluting with hexanes/AcOEt 9:1 afforded pure 5fa, as an oil (64.9 mg, 60\%).

\footnotetext{
${ }^{3}$ Guss, C. O.; Jules, L. H. J. Am. Chem. Soc. 1950, 72, 3462.
} 
${ }^{1} \mathrm{H}$ NMR (250 MHz, $\left.\mathrm{CDCl}_{3}\right) \delta 2.07$ (s, 3H); 3.58 (s, 3H); 3.77 (s, 3H); 4.22-4.38 (m, 3H); 4.70-4.78 (m, 1H); 6.00 (d, 1H, J=2.0 Hz); 6.13 (d, 1H, J=2.0 Hz); 7.09-7.15 (m, 2H); 7.20-7.31 (m, 3H).

${ }^{13} \mathrm{C}$ NMR (62.5 MHz, $\mathrm{CDCl}_{3}$ ) $\delta$ 20.8, 48.6, 55.3, 55.5, 65.1, 88.4, 89.4, 91.9, 108.1, 126.8, 127.2, 128.6, 142.3, 157.2, 161.3, 162.3, 170.2 .

Anal. Calcd. For $\mathrm{C}_{19} \mathrm{H}_{14} \mathrm{O}$ : C, 69.50\%; H, 6.14\%. Found: C, 69.87\%; H, 5.89\%.

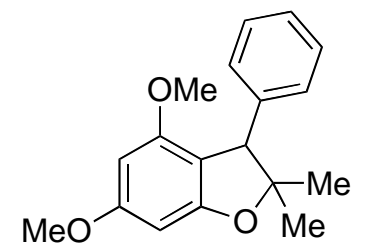

\section{4,6-Dimethoxy-2,2-dimethyl-3-phenyl-2,3-dihydro benzofuran (4qa) (table 2, entry 9).}

Using the general procedure, a solution of tris-(3,5dimethyoxyphenyl) borate (1a) (705 mg, $1.5 \mathrm{mmol}$ ) in $\mathrm{CH}_{2} \mathrm{Cl}_{2}$ $(1.0 \mathrm{~mL})$ was added at $\mathrm{rt}$ under argon to a solution of 1,2-epoxy-2-methyl-1-phenylpropane (2q) (148 $\mathrm{mg}, 1.0 \mathrm{mmol})$ in $\mathrm{CH}_{2} \mathrm{Cl}_{2}(0.5 \mathrm{~mL})$. The mixture was allowed to react for 18 hours at $-40^{\circ} \mathrm{C}$. The reaction was then quenched and the solvent was evaporated under a vacuum. To the crude mixture dissolved in toluene $(18.5 \mathrm{~mL})$ was then added with $p$-TsOH (28.5 mg, $0.15 \mathrm{mmol}$ ) and it was allowed to react for 3 hours at $100^{\circ} \mathrm{C}$. The mixture was quenched with aqueous $\mathrm{NaHCO}_{3}$ saturated solution, dried over $\mathrm{MgSO}_{4}$ and filtered. Column chromatography eluting with hexanes/Et $\mathrm{E}_{2} \mathrm{O}$ 9:1 afforded pure $\mathbf{4 q a}$, as a solid (156.2 $\mathrm{mg}, 55 \%)$. M.p= 86-89 ${ }^{\circ} \mathrm{C}$.

${ }^{1} \mathrm{H}$ NMR (250 MHz, $\mathrm{CDCl}_{3}$ ) $\delta 0.97$ (s, 3H); 1.53 (s, 3H); 3.60 (s, 3H); 3.80 (s, 3H); 4.18 (s, 1H); 6.02 (s, 1H); 6.11 (s, 1H); 6.90-7.10 (m, 2H); 7.15-7.28 (m, 3H).

${ }^{13} \mathrm{C}$ NMR (62.5 MHz, $\left.\mathrm{CDCl}_{3}\right) \delta$ 24.5, 29.4, 55.3, 55.5, 55.6, 88.3, 90.4, 91.3, 109.4, 126.6, 127.0, 127.3, 140.4, 157.3, 160.7, 162.0

Anal. Calcd. For $\mathrm{C}_{19} \mathrm{H}_{14} \mathrm{O}$ : C, 76.03\%; H, 7.09\%. Found: C, 75.87\%; H, 6.89\%.

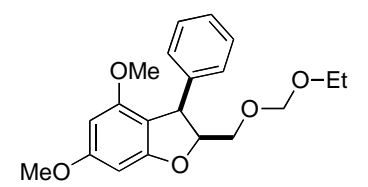

$\left(2 S^{*}, 3 R^{*}\right)-2,3-D i h y d r o-4,6-d i m e t h o x y-2-$

(ethoxymethoxy)methyl-3-phenyl-benzofuran (4ja) (Table

2, entry 10). Using the general procedure, triphenylphosphine (251.8 mg, $0.96 \mathrm{mmol}$ ) and diethylazodicarboxylate (125.3 mg, $114 \mu \mathrm{L}, 0.72 \mathrm{mmol}$ ) were added to a solution of 2-((1R*,2R*)-3-(ethoxymethoxy)-2-hydroxy-1phenylpropyl)-3,5-dimethoxy phenol (3hi) (173 mg, $0.48 \mathrm{mmol}$ ) in anhydrous THF $(1.9 \mathrm{~mL})$. The mixture was allowed to react for 2 hours at rt. Column chromatography eluting with hexanes/AcOEt 8:2, afforded pure 4ja (128.8 mg, 78\%), as an oil. ${ }^{1} \mathrm{H}$ 
NMR (250 MHz, $\left.\mathrm{CDCl}_{3}\right) \delta 1.13(\mathrm{t}, 3 \mathrm{H}, J=7.1 \mathrm{~Hz}) ; 3.25-3.41(\mathrm{~m}, 2 \mathrm{H}) ; 3.50$ (q, 2H, $J=7.1 \mathrm{~Hz})$; 3.56 (s, 3H); 3.76 (s, 3H); 4.49- 4.58 (m, 3H); 4.97- 5.25 (m, 1H); 6.00 (d, $1 \mathrm{H}, J=2.0 \mathrm{~Hz}$ ); 6.17 (d, 1H, $J=2.0 \mathrm{~Hz}) ; 6.90-7.05$ (m, 2H); 7.12- 7.25 (m, 3H). ${ }^{13} \mathrm{C}$ NMR (62.5 MHz, $\mathrm{CDCl}_{3}$ ) $\delta$ 15.0, 47.6, 55.3, 55.5, 63.2, 67.9, 86.2, 88.5, 91.9, 95.2, 109.9, 126.9, 128.1, 128.3, 138.6, 156.8, 161.6, 162.2.

Anal. Calcd. For $\mathrm{C}_{20} \mathrm{H}_{24} \mathrm{O}_{5}$ : C, 69.75\%; H, 7.02\%. Found: C, 69.89\%; H, 6.89\%.

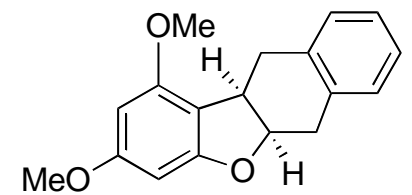

\section{Compound 4ka (table 2, entry 11).}

Using the general procedure, to a solution of $\left(1 S^{*}, 2 R^{*}\right)-1$ (2-hydroxy-4,6-dimethoxy phenyl)-1,2,3,4tetahydronaphtalen-2-ol (3ka) (143.0 mg, $0.48 \mathrm{mmol})$ in anhydrous THF (1.92 mL) were added triphenylphosphine (251.8 $\mathrm{mg}, 0.96 \mathrm{mmol}$ ) and diethylazodicarboxylate (125.3 mg, $0.72 \mathrm{mmol}$ ). The mixture was allowed to react for 2 hours at rt. Column chromatography eluting with hexanes/AcOEt 8:2 afforded pure 4ka (53.8 mg, 40\%), as an oil. ${ }^{1} \mathrm{H}$ NMR (250 MHz, $\left.\mathrm{CDCl}_{3}\right) \delta 1.72-1.90$ (m, 1H); 2.36-2.48 (m, 1H); 2.432.56 (m, 1H); 2.83-3.00 (m, 1H); 3.73 (s, 1H); 3.80 (s, 3H); 4.67 (d, 1H, J= $8.25 \mathrm{~Hz}$ ); 5.21-5.30 (m, 1H); 5.95 (d, 1H, J=2.0 Hz); 6.03 (d, 1H, J=2.0 Hz); 7.03-7.28 (m, 3H); 7.68 (d, $1 \mathrm{H}, J=7.25 \mathrm{~Hz}) .{ }^{13} \mathrm{C}$ NMR $\left(62.5 \mathrm{MHz}, \mathrm{CDCl}_{3}\right) \delta 24.3,27.9,42.3,55.2$, 55.5, 83.1, 88.2, 91.4, 110.2, 125.9, 126.0, 128.2, 130.2, 136.2, 137.7, 157.3, 161.6, 161.7. Anal. Calcd. For $\mathrm{C}_{19} \mathrm{H}_{20} \mathrm{O}_{2}$ : C, 81.40\%; H, 7.19\%. Found: C, 81.33\%; H, $7.21 \%$.<smiles>COc1cc(O)c(C2=C(c3ccccc3)CCCC2)c(OC)c1</smiles>
2-(2-Phenylcyclohex-1-enyl)-3,5-dimethoxyphenol (6). Using the general procedure, triphenylphosphine (141.6 mg, $0.40 \mathrm{mmol}$ ) and diethylazodicarboxylate (69.6 $\mathrm{mg}, 0.50 \mathrm{mmol}$ ) were added to a solution of compound 3ha (88.6 mg, $0.27 \mathrm{mmol}$ ) in anhydrous THF (1.1 mL). The mixture was allowed to react for 5 hours at $\mathrm{rt}$ and then the solvent was removed in vacuo. Column chromatography eluting with hexanes/AcOEt 9:1 afforded pure 6 (50.2 mg, 60\%), as an oil. ${ }^{1} \mathrm{H}$ NMR (250 MHz, $\left.\mathrm{CDCl}_{3}\right) \delta$ 1.62-1.85 (m, 4H); 2.12-2.25 (m, 2H); 2.42-2.71 (m, 2H); 3.62 (s, 3H); 3.77 (s, 3H); $5.36 \mathrm{ArOH}$ (br, s, 1H); 6.07 (d, 1H, J=2.3 Hz); 6.17 (d, 1H, J=2.3 Hz); 7.057.38 (m, 5H). ${ }^{13} \mathrm{C}$ NMR (62.5 MHz, $\left.\mathrm{CDCl}_{3}\right) \delta$ 25.8, 27.4, 33.0 (2 C); 55.2, 55.7, 91.7, 92.3, 111.3, 123.0, 126.2, 127.8, 128.07, 141.3, 150.0, 153.6, 158.0, 160.5 
Anal. Calcd. For $\mathrm{C}_{20} \mathrm{H}_{22} \mathrm{O}_{3}$ : C, 77.39\%; H, 7.14\%. Found: C, 77.64\%; H, 7.04\%.

TABLE 3. Additional Results of Cyclodehydration of Hydroxyphenols to 2,3-Dihydrobenzofuranes.

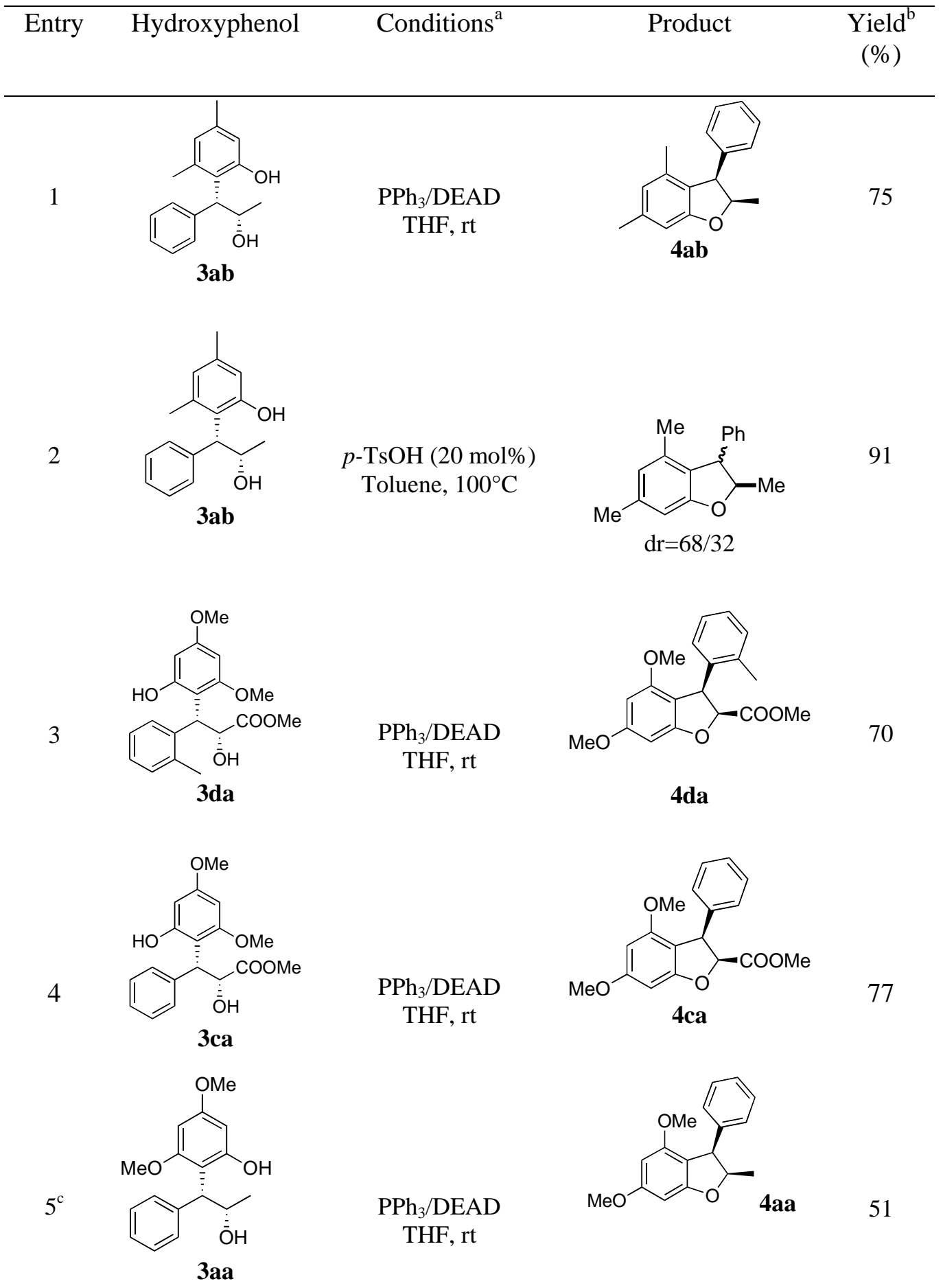

\footnotetext{
${ }^{a}$ All reactions were carried out in accordance with general procedures. ${ }^{b}$ Isolated yield after chromatography on silica gel. ${ }^{\mathrm{c}}$ Compound $3 \mathbf{a a}$ was not isolated but directly cyclized to $4 \mathbf{a a}$ using the Mitsunobu-type cyclodehydration.
} 


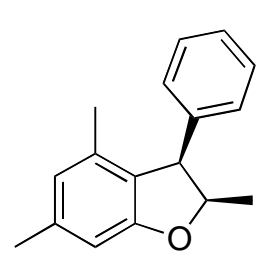

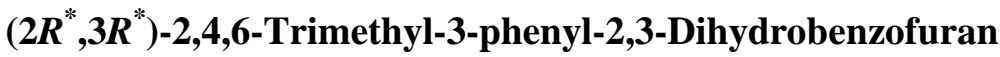
(4ab) (table 3, entry 1).

Using the general procedure, to a solution of $2-\left(\left(1 R^{*}, 2 S^{*}\right)-2-\right.$

hydroxy-1-phenylpropyl)-3,5-dimethylphenol (3ab) (59.5 mg, 0.25

$\mathrm{mmol})$ in anhydrous THF $(1.00 \mathrm{~mL})$ were added triphenylphosphine (131.2 mg, 0.50 mmol) and diethylazodicarboxylate $(66.1 \mathrm{mg}, 0.38 \mathrm{mmol})$. The mixture was allowed to react for 1 hour at rt. The product (containing $3.6 \%$ of trans-stereoisomer as determined by GC) was isolated (Yield=75 \%) by column chromatography eluting with hexanes/Et $2 \mathrm{O} 9: 1$, as an oil.

${ }^{1} \mathrm{H}$ NMR $\left(\mathrm{CDCl}_{3}, 200 \mathrm{MHz}\right) \delta 1.09$ (d, 3H, J=6.5 Hz); 1.93 (s, 3H); 2.32 (s, 3H); 4.35 (d, 1H, J= 8.2 Hz); 4.95-5.11 (m, 1H); 6.53 (s, 1H); 6.57 (s, 1H); 6.90-7.01 (m, 2H); 7.15-7.40 (m 3H).

${ }^{13} \mathrm{C} \mathrm{NMR}\left(\mathrm{CDCl}_{3}, 50 \mathrm{MHz}\right) \delta 17.0,18.4,21.6,51.0,83.2,107.6,123.0,126.8,127.6$, 127.8, 128.3, 128.9, 135.1, 138.8, 139.0, 160.0.

MS (relative intensity) 238 ( $\left.\mathrm{M}^{+}, 100\right), 223$ (85), 209 (20), 195 (40), 178 (16), 115 (21). Anal. Calcd. For $\mathrm{C}_{17} \mathrm{H}_{18} \mathrm{O}$ : C, 85.67\%; H, 7.61\%. Found: C, 85.90\%; H, 7.55\%.

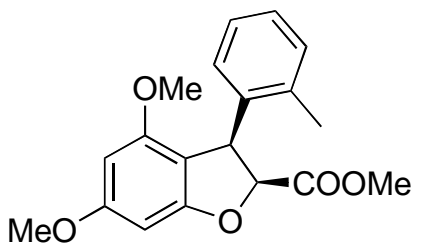

methyl $\left(2 S^{*}, 3 R^{*}\right)$-Methyl 4,6-dimethoxy-3-(2-methylphenyl)2,3-dihydrobenzofuran-2-carboxylate (4da) (table 3, entry 3).

Using the general procedure, to a solution of $\left(2 R^{*}, 3 R^{*}\right)-$ 2-hydroxy-3-(2-hydroxy-4,6-dimethoxyphenyl)-3-(2-

methylphenyl)propanoate (3da) (126.7 mg, $0.37 \mathrm{mmol})$ in anhydrous THF (1.5 mL) were added triphenylphosphine (194.1 $\mathrm{mg}, 0.74 \mathrm{mmol})$ and diethylazodicarboxylate (52.2 $\mathrm{mg}, 0.3 \mathrm{mmol}$ ). The mixture was allowed to react for 1 hour at rt. The product was isolated (Yield $=70 \%$ ) by column chromatography eluting with hexanes/AcOEt 8:2, as a solid. M.p $=113-114^{\circ} \mathrm{C}$.

${ }^{1} \mathrm{H}$ NMR (CDCl, $\left.250 \mathrm{MHz}\right) \delta 2.47$ (s, 3H); 3.23 (s, 3H); 3.60 (s, 3H); 3.80 (s, 3H); 5.13 (d, 1H, J= $9.5 \mathrm{~Hz}) ; 5.49$ (d, 1H, $J=9.5 \mathrm{~Hz}$ ); 6.03 (d, 1H, $J=1.5 \mathrm{~Hz}) ; 6.80$ (d, 1H, $J=7.2 \mathrm{~Hz}) ; 6.96-7.24(\mathrm{~m}, 3 \mathrm{H})$. 
${ }^{13} \mathrm{C} \mathrm{NMR}\left(\mathrm{CDCl}_{3}, 62.5 \mathrm{MHz}\right) \delta$ 19.8, 44.5, 51.5, 55.4, 55.6, 85.2, 88.5, 92.4, 108.7, 125.9, 126.9, 127.7, 129.6, 135.9, 136.9, 156.5, 161.2, 162.3, 168.9.

Anal. Calcd. For $\mathrm{C}_{19} \mathrm{H}_{14} \mathrm{O}$ : C, 69.50\%; H, 6.14\%. Found: C, 69.87\%; H, 6.09\%.

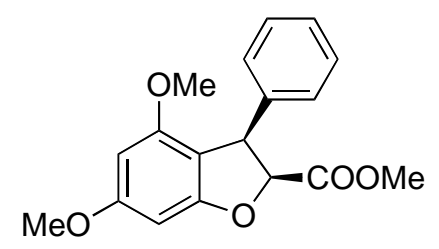

$\left(2 S^{*}, 3 R^{*}\right)$-Methyl 4,6-dimethoxy-3-phenyl-2,3-dihydro benzofuran-2-carboxylate (4ca) (table 3, entry 4).

Following the procedure $\mathbf{A}$ described above, to a solution of $\quad\left(2 R^{*}, 3 R^{*}\right)$-methyl 2-hydroxy-3-(2-hydroxy-4,6dimethoxyphenyl)-3-phenylpropanoate (3ca) (containing $13 \%$ of $2 S^{*}, 3 S^{*}$ stereoisomer) (66.8 mg, $0.20 \mathrm{mmol})$ in anhydrous THF $(0.8 \mathrm{~mL})$ were added triphenylphosphine (104.9 mg, $0.4 \mathrm{mmol}$ ) and diethylazodicarboxylate (52.2 mg, 0.3 $\mathrm{mmol}$ ). The mixture was allowed to react for 1 hour at rt. The product (containing $13 \%$ of the trans-diastereoisomer 5ca) was isolated (Yield=77\%) by column chromatography eluting with hexanes/AcOEt 8:2, as a solid.

${ }^{1} \mathrm{H} \mathrm{NMR}\left(\mathrm{CDCl}_{3}, 250 \mathrm{MHz}\right) \delta 3.27$ (s, 3H); 3.58 (s, 3H); 3.80 (s, 3H); 4.79 (d, 1H, J= $9.25 \mathrm{~Hz}$ ); 5.43 (d, 1H, J= 9.25 Hz); 6.03 (d, 1H, $J=2.0 \mathrm{~Hz}$ ); 6.24 (d,1H, $J=2.0 \mathrm{~Hz}$ ); 6.95-7.05 (m, 2H); 7.13- $7.23(\mathrm{~m}, 3 \mathrm{H})$.

${ }^{13} \mathrm{C} \mathrm{NMR}\left(\mathrm{CDCl}_{3}, 62.5 \mathrm{MHz}\right) \delta$ 49.2, 51.6, 55.4, 55.5, 85.8, 88.6, 92.5, 108.2, 127.2, 127.9, 128.2, 138.4, 156.8, 161.1, 162.4, 168.7.

Anal. Calcd. For $\mathrm{C}_{19} \mathrm{H}_{14} \mathrm{O}$ : C, 68.78\%; H, 5.77\%. Found: C, 69.07\%; H, 5.39\%.

Representative signals of compound 5ca: ${ }^{1} \mathrm{H}$ NMR (250 MHz, $\left.\mathrm{CDCl}_{3}\right) \delta 4.71$ (d, $1 \mathrm{H}$, $J=4.0 \mathrm{~Hz}), 4.98$ (d, $1 \mathrm{H}, J=4.0 \mathrm{~Hz}) .{ }^{13} \mathrm{C} \mathrm{NMR}\left(\mathrm{CDCl}_{3}, 62.5 \mathrm{MHz}\right) \delta 50.3,87.9$.

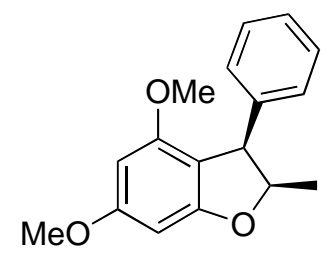

(2 $R^{*}, 3 R^{*}$ )-4,6-Dimethoxy-2-methyl-3-phenyl-2,3-dihydro benzofuran (4aa) (table 3, entry 5 ).

Using the general procedure for the stereoselective ring-opening of aryl epoxides, a solution of tris (3,5-dimethoxyphenyl) borate (1a) (705 mg, $1.5 \mathrm{mmol})$ in $\mathrm{CH}_{2} \mathrm{Cl}_{2}(1.0 \mathrm{~mL})$ was added at rt under argon to a solution of trans- $\beta$-methyl-styrene (2a) (134 mg, $1.0 \mathrm{mmol}$ ) in $\mathrm{CH}_{2} \mathrm{Cl}_{2}(0.5 \mathrm{~mL})$. The mixture was allowed to react for 18 hours at $-78^{\circ} \mathrm{C}$. The reaction was then quenched and the solvent was evaporated under a vacuum. To the crude mixture, containing $27 \%$ of $O$ alkylated product, dissolved in THF $(2.9 \mathrm{~mL})$ were then added triphenylphosphine (382.9 mg, $1.46 \mathrm{mmol}$ ) and diethylazodicarboxylate (189.7 mg, $1.09 \mathrm{mmol})$. The 
mixture was allowed to react for 2 hours at rt. The purification by flash column chromatography eluting with hexanes/Et $2 \mathrm{O} 9: 1$ afforded $\left(2 R^{*}, 3 R^{*}\right)-4,6$-dimethoxy-2methyl-3-phenyl-2,3-dihydrobenzofuran (4aa) (contaminated with 19\% of the transdiastereoisomer 5aa, see below), as an oil (137.7 mg, 51\%).

${ }^{1} \mathrm{H}$ NMR $\left(\mathrm{CDCl}_{3}, 250 \mathrm{MHz}\right) \delta 1.02$ (d, 3H, J=6.3 Hz); 3.60 (s, 3H); 3.78 (s, 3H); 4.38 (d, 1H, J=8.3 Hz); 4.93-5.10 (m, 1H); 6.01 (s, 1H); 6.13 (s, 1H); 6.92 (d, 2H, $J=7.3 \mathrm{~Hz}$ ); 7.10-7.27 (m, 3H).

${ }^{13} \mathrm{C} \mathrm{NMR}\left(\mathrm{CDCl}_{3}, 62.5 \mathrm{MHz}\right) \delta 16.8,49.3,55.4,55.5,84.4$, 88.3, 91.6, 126.6, 127.2, 128.4, 139.3, 156.8, 161.9 (2C).

Anal. Calcd. For $\mathrm{C}_{17} \mathrm{H}_{18} \mathrm{O}_{3}$ : C, 75.53\%; H, 6.71\%. Found: C, 75.47\%; H, 6.49\%.

Representative signals of $\left(2 S^{*}, 3 R^{*}\right)$-stereoisomer (trans-5aa):

${ }^{1} \mathrm{H}$ NMR (250 MHz, $\left.\mathrm{CDCl}_{3}\right) \delta 4.11$ (d, 1H, $J=5.0 \mathrm{~Hz}$ ); 4.60-4.70 (m, 1H). 